\title{
Why Don't We Move Faster? Parkinson's Disease, Movement Vigor, and Implicit Motivation
}

\author{
Pietro Mazzoni, Anna Hristova, and John W. Krakauer \\ Motor Performance Laboratory, Department of Neurology, Columbia University College of Physicians and Surgeons, New York, New York 10032
}

\begin{abstract}
People generally select a similar speed for a given motor task, such as reaching for a cup. One well established determinant of movement time is the speed-accuracy trade-off: movement time increases with the accuracy requirement. A second possible determinant is the energetic cost of making a movement. Parkinson's disease (PD), a condition characterized by generalized movement slowing (bradykinesia), provides the opportunity to directly explore this second possibility. We compared reaching movements of patients with PD with those of control subjects in a speed-accuracy trade-off task comprising conditions of increasing difficulty. Subjects completed as many trials as necessary to make 20 movements within a required speed range (trials to criterion, $N_{c}$ ). Difficulty was reflected in endpoint accuracy and $N_{c}$. Patients were as accurate as control subjects in all conditions (i.e., PD did not affect the speed-accuracy trade-off). However, $N_{c}$ was consistently higher in patients, indicating reluctance to move fast although accuracy was not compromised. Specifically, the dependence of $N_{c}$ on movement energy cost (slope $S_{N}$ ) was steeper in patients than in control subjects. This difference in $S_{N}$ suggests that bradykinesia represents an implicit decision not to move fast because of a shift in the cost/benefit ratio of the energy expenditure needed to move at normal speed. $S_{N}$ was less steep, but statistically significant, in control subjects, which demonstrates a role for energetic cost in the normal control of movement speed. We propose that, analogous to the established role of dopamine in explicit reward-seeking behavior, the dopaminergic projection to the striatum provides a signal for implicit "motor motivation."
\end{abstract}

Key words: movement time; speed-accuracy trade-off; bradykinesia; dopamine; motor planning; reward

\section{Introduction}

Everyday observation indicates that movement time (MT) is controlled: movements are performed at a "natural" speed that is remarkably uniform for a given task. When watching a video clip of a person reaching for an object, lighting a match, or gesticulating, we can tell whether the clip has been sped up or slowed down. MT has long been known to affect endpoint accuracy (Woodworth, 1899; Fitts, 1954; Schmidt et al., 1979). We recently introduced a model that successfully predicts MT by minimizing it for a given desired accuracy (Tanaka et al., 2006). We acknowledged, however, that factors other than the speed-accuracy trade-off may influence MT: everyday movements could be performed faster than usual without loss of accuracy. One factor may be energy expenditure. Indeed, a recent model of trajectory control included energetic cost in its optimization (Todorov and Jordan, 2002).

Parkinson's disease (PD) provides the unique opportunity to test hypotheses about MT selection experimentally rather than through modeling. A cardinal feature of PD is generalized move-

\footnotetext{
Received Jan. 19, 2007; revised May 23, 2007; accepted May 24, 2007.

This work was supported by the Parkinson's Disease Foundation, the Lowenstein Foundation, and National Institutes of Health Grant NS048099. We thank Dr. Toni S. Pearson for critical comments on this manuscript, Dr. Eric Zarahn for assistance with analysis, Dr. Claude Ghez for insightful discussion, Joseph W. Schumacher for technical assistance, and Dr. Robert Sainburg for sharing custom computer software.

Correspondence should be addressed to Dr. Pietro Mazzoni, Department of Neurology, Columbia University College of Physicians and Surgeons, 710 West 168th Street, Box NI-175, New York, NY 10032. E-mail: pm125@columbia.edu.

DOI:10.1523/JNEUROSCI.0264-07.2007

Copyright $\odot 2007$ Society for Neuroscience $\quad$ 0270-6474/07/277105-12\$15.00/0
}

ment slowing (bradykinesia), which critically cannot be explained by an intrinsic limitation in execution (England and Schwab, 1961), but rather is a problem of scaling speed to movement distance (Hallett and Khoshbin, 1980; Berardelli et al., 2001). A proposed explanation for this scaling deficit is that bradykinesia is a compensatory response: patients slow down because of a loss of accuracy at normal speeds (Montgomery and Nuessen, 1990; Sheridan and Flowers, 1990; Phillips et al., 1994).

We hypothesized instead that a major determinant of MT is the energetic cost of a movement. This hypothesis can be tested directly in PD, because it predicts that bradykinesia is caused by a shift in the cost/benefit ratio of moving fast. Showing such a shift would raise the novel possibility that low-level movement parameters, such as MT, can fit within the framework of cost and reward (Schultz, 2006). In free-operant experiments, a primary variable is response vigor (e.g., how frequently a rat presses a lever for food). A recent model proposes that response vigor is determined by balancing costs and benefits of quick responding, and that this balance is shifted by motivational state and signaled by dopamine levels (Niv et al., 2007). We introduce the idea that, just as dopamine from midbrain ventral tegmental projections to the frontal cortex determines the likelihood of a reinforced behavior (response vigor), dopamine from the substantia nigra to the neostriatum regulates the likelihood of moving at a certain speed ("movement vigor").

We tested patients with PD in a speed-accuracy task comprising conditions of increasing difficulty. In each condition, subjects completed as many trials as necessary to make 20 movements 
Table 1. Characteristics of study participants

\begin{tabular}{|c|c|c|c|c|c|c|c|c|c|}
\hline $\begin{array}{l}\text { Subject } \\
\text { code }\end{array}$ & Group & Age & Handedness & Sex & $\begin{array}{l}\text { Disease duration } \\
\text { (years) }\end{array}$ & $\begin{array}{l}\text { Hoehn-Yahr } \\
\text { stage }(0-5)\end{array}$ & $\begin{array}{l}\text { Motor UPDRS } \\
\text { score }(0-108)\end{array}$ & $\begin{array}{l}\text { Dominant arm } \\
\text { rigidity }(0-4)\end{array}$ & $\begin{array}{l}\text { DUEBR } \\
(0-16)\end{array}$ \\
\hline P1 & PD & 64 & RH & $M$ & 5.3 & 2 & 22 & 2 & 5 \\
\hline P2 & PD & 68 & RH & $M$ & 9.8 & 2 & 26 & 1 & 4 \\
\hline P3 & PD & 68 & RH & $M$ & 3.7 & 2 & 20 & 3 & 8 \\
\hline P4 & PD & 55 & $\mathrm{RH}$ & $\mathrm{F}$ & 4.2 & 1 & 10 & 1 & 4 \\
\hline P5 & PD & 71 & $\mathrm{RH}$ & M & 5.8 & 2 & 15 & 2 & 5 \\
\hline P6 & PD & 70 & $\mathrm{LH}$ & $\mathrm{F}$ & 7.4 & 2 & 30 & 2 & 7 \\
\hline P7 & PD & 64 & $\mathrm{LH}$ & M & 2.5 & 2 & 22 & 2 & 7 \\
\hline Mean \pm SD & & $66 \pm 9$ & & & $5.5 \pm 2.5$ & & $21 \pm 7$ & $1.8 \pm 0.8$ & $5.7 \pm 1.6$ \\
\hline $\mathrm{C} 1$ & $\mathrm{Ctl}$ & 57 & $\mathrm{RH}$ & $M$ & & & & & \\
\hline $\mathrm{C} 2$ & $\mathrm{Ctl}$ & 68 & $\mathrm{RH}$ & $\mathrm{F}$ & & & & & \\
\hline C 3 & $\mathrm{Ctl}$ & 70 & $\mathrm{LH}$ & M & & & & & \\
\hline C4 & $\mathrm{Ctl}$ & 43 & $\mathrm{RH}$ & $\mathrm{F}$ & & & & & \\
\hline$C 5$ & $\mathrm{Ctl}$ & 64 & $\mathrm{RH}$ & $F$ & & & & & \\
\hline C6 & $\mathrm{Ctl}$ & 68 & $\mathrm{RH}$ & $\mathrm{F}$ & & & & & \\
\hline C7 & $\mathrm{Ctl}$ & 66 & $\mathrm{RH}$ & $\mathrm{F}$ & & & & & \\
\hline Mean \pm SD & & $62 \pm 9$ & & & & & & & \\
\hline$p$ & & 0.42 & 1.00 & 0.29 & & & & & \\
\hline
\end{tabular}

Motor UPDRS, Total score of the motor examination section of the UPDRS (items 18-31); DUEBR, dominant upper extremity bradykinesia and rigidity (total of UPDRS items $22-25$ for the arm tested); RH, right hand; LH, left hand; M, male; $\mathrm{F}$, female; $\mathrm{Ctl}$, control. The numbers in parentheses indicate the range of possible scores for each measure. $p$ value data: two-sample $t$ test for age; Fisher's exact test for handedness and sex.

within a required speed range (trials to criterion, $N_{c}$ ). Conditions differed in speed requirement and target distance. Difficulty was reflected in endpoint accuracy and $N_{c}$. $N_{c}$ is analogous to the number of lever presses per unit time for a rat in a free-operant paradigm. If bradykinesia is attributable to decreased movement vigor rather than compensation, then we expected patients to show a higher sensitivity of $N_{c}$ to condition without a concomitant decrease in accuracy.

\section{Materials and Methods}

\section{Subjects}

Seven patients with idiopathic PD and seven age-matched control subjects participated in the study (Table 1). The mean age was 66 years (range, 55-71) for patients and 62 years (range, 43-70) for control subjects. For patients, the mean disease duration was 5.5 years (range, 2.59.8). Clinical severity was measured by the motor component of the Unified Parkinson's Disease Rating Scale (UPDRS) (Fahn et al., 1987), of which possible values range from 0 (normal) to 108 (maximum severity). The patients' mean motor UPDRS score was 21 (range, 10-30). All patients had clinically detectable rigidity and bradykinesia in the upper extremity tested (Table 1). All subjects were free of musculoskeletal disease. Control subjects had no neurological disease, and patients had no neurological disease other than PD. The diagnosis of PD was confirmed by a neurologist with specialty training in movement disorders (P.M.), based on review of the clinical history, a neurological examination, and response to dopaminergic treatment. P.M. also performed a UPDRS examination on the day of testing. All subjects were free of dementia as assessed by a Mini-mental Status Examination (Folstein et al., 1975), on which all subjects scored 30 of 30 . The dominant arm, which was tested in this study, was affected in all patients. Four of the PD patients were taking dopamine agonist medications at the time of testing. All subjects gave written informed consent (consistent with the Declaration of Helsinki) to participate in the study, which was approved by the Columbia University Institutional Review Board.

\section{Apparatus}

Figure 1 illustrates the experimental setup. Subjects sat at a glass-surface table (Fig. $1 A$ ) with their dominant arm supported on a lightweight sled that hovered on air cushions created by compressed-air jets, which allowed frictionless planar motion of the upper arm and forearm (Sainburg et al., 1999). Motion of the trunk and wrist was prevented with mechanical splints. Seat height was individually adjusted so that shoulder, elbow, and hand were in the same plane, parallel to the table surface. Subjects viewed the reflection of a computer display [liquid crystal dis-

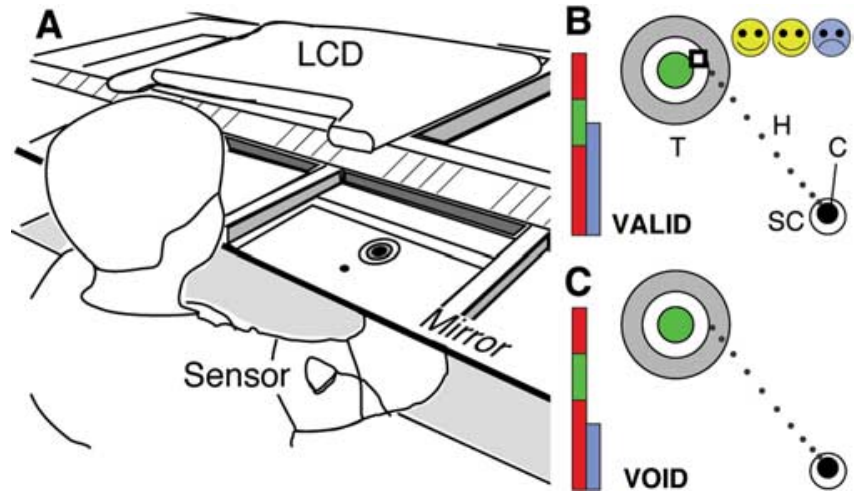

Figure 1. Experimental apparatus and motor task. $A$, Subject sits with right arm supported over glass surface and looks in the mirror, which reflects the LCD. The upper arm magnetic sensor is visible (forearm sensor is hidden by mirror). $\boldsymbol{B}$, Valid trial display. Before the trial, the subject sees start circle $S C$, target $T$, cursor $C$, and the vertical bar indicating required speed range in green. During movement, $\mathrm{C}$ disappears and the hand follows hand path $\mathrm{H}$ (not visible to subject). At the end of the trial, the blue vertical bar indicates peak velocity of the movement just made, the white square indicates movement endpoint, and smiley faces appear. $\mathbf{C}$, Void trial display. Peak velocity is outside the required range, and the white square and smiley faces do not appear.

play (LCD)] in a mirror suspended halfway between the subject's hand and the LCD, so that the virtual image of the display in the mirror was in the plane of arm motion (veridical display). The mirror blocked the subjects' view of their arm and hand. We used a "Flock of Birds" magnetic system (Ascension Technology, Burlington, VT) to record hand and arm position: a transmitter positioned $23 \mathrm{~cm}$ below the glass surface bathed the workspace in an alternating magnetic field, and two sensors (one attached to the sled and one attached to the upper arm) recorded position and orientation of the two arm segments at $120 \mathrm{~Hz}$ with a resolution of $\pm 1 \mathrm{~mm}$. The experimental computer (Power Macintosh G4; Apple, Cupertino, CA) converted this information on-line into hand, elbow, and shoulder positions using custom-written routines (courtesy of Dr. Robert L. Sainburg, Penn State University, State College, PA) developed in RealBasic (Real Software, Austin, TX). The computer used real-time hand-position information to control the visual display and to provide visual and auditory feedback. 


\section{Task}

Subjects made planar aiming movements of the arm and hand to a visual target. Before each trial, subjects moved their arm to guide a screen cursor indicating their hand's position (tip of middle finger) into a "start" circle of $1 \mathrm{~cm}$ radius (Fig. $1 \mathrm{~B}$ ). The cursor became visible only when the hand was within $1 \mathrm{~cm}$ of the perimeter of the start circle, thus removing intertrial visual feedback. Each trial started after the subject maintained the cursor inside the start circle for $750 \mathrm{~ms}$, at which point a "bull's eye" target with a center circle of $1 \mathrm{~cm}$ radius $(\mathrm{T})$ appeared in the $135^{\circ}$ ("northwest") direction (Fig. $1 \mathrm{~B}$ ), at a distance dictated by the task condition. The direction was $45^{\circ}$ ("northeast") for left-handed subjects. The instruction was to move the hand, when ready, into the target in a single motion. The cursor disappeared when the hand left the start circle, and the movement proceeded without on-line visual guidance. The computer continuously monitored the hand's distance from the start circle and its instantaneous tangential velocity. When the hand's distance from the start circle stopped increasing (defined as the endpoint of the movement), the computer compared the hand's peak velocity to the required range: if it was within this range, the trial was labeled a "valid" trial, and visual feedback was instantaneously given in the form of a stationary white square, which appeared at the location of the endpoint for $2 \mathrm{~s}$ (Fig. $1 B)$. Schematic smiling face drawings were shown to reward spatial accuracy: three faces if the white square was in the smallest of the concentric circles of the target; two faces for the middle circle; one face for the outer circle; and none if the square was outside the target (Fig. $1 B$ ). If peak velocity was not reached, the trial was labeled "void," and no visual feedback about spatial accuracy was given (no white square, no smiley faces) (Fig. 1C). Instead, the speech synthesizer of the computer uttered the phrase "Too slow" or "Too fast." Between trials, a vertical blue bar showed the peak velocity of the previous trial, alongside a vertical bar that indicated in green the range of required peak velocity and in red speeds outside this range (Fig. $1 B, C$ ). Two seconds after the end of each trial, a tone instructed subjects to move their arm back to the start circle to start the next trial.

Subjects were instructed that the goal was to place the white square as close as possible to the center of the target with a movement whose speed was dictated by the computer. Subjects were reminded, before each block, to start each movement only when ready: there was no "reactiontime" requirement. Because the white square appeared when the hand's distance from the start circle reached its first maximum, spatial accuracy was based on a single movement, and additional submovements were irrelevant to the task.

Familiarization. To gain familiarity with the apparatus, subjects made 10 movements under continuous visual guidance: trials occurred as described above (see Task), except that the cursor remained visible throughout the trial. The $12 \mathrm{~cm}$ target and the slow speed requirements (see below) were used for familiarization.

Speed-accuracy conditions. After familiarization, subjects were told that they had to perform 20 valid movements in each condition and that movements outside the required speed range (void trials) did not count. Each condition was defined by a target distance $(6,12,16 \mathrm{~cm})$ and a range of required peak velocity [slow (S), 17-37 cm/s; medium (M), 37-57 $\mathrm{cm} / \mathrm{s}$; fast (F), $57-77 \mathrm{~cm} / \mathrm{s}$; very fast (VF), $77-97 \mathrm{~cm} / \mathrm{s}]$ and was presented as a block in the following sequence: $12 \mathrm{~S}, 12 \mathrm{M}, 12 \mathrm{~F} ; 16 \mathrm{M}, 16 \mathrm{~F}, 16 \mathrm{VF} ; 6 \mathrm{M}$. A condition block ended when the subject performed 20 movements within the speed range required for that condition. Subjects were instructed that they could rest their arm any time they wanted simply by waiting before placing the cursor in the start circle and that there was no time limit on the testing session. The condition blocks were presented in the same order for all subjects.

\section{Data analysis}

We analyzed hand position data using custom routines within the IGOR analysis software package (Wavemetrics, Lake Oswego, OR). Position time series were filtered using a smoothing spline routine (smoothing factor, 0.005) (Reinsch, 1967) and differentiated to yield tangential velocity, acceleration, and jerk. Left-handed subjects performed the task with their left arm, and targets were placed symmetrically opposite, across the midline, to those used for right-handed subjects. Raw position data for left-handers was then transposed across the midline and combined with right-handers' data in the analysis.

Trajectory variables. Peak velocity was identified as the first zero crossing of tangential acceleration after the hand reached a distance from the start circle that was $>60 \%$ of the distance from start circle to the target. From the time of peak velocity, the velocity trace was scanned backward in time to identify the time when velocity crossed the $2 \mathrm{~cm} / \mathrm{s}$ value, which was defined as the movement start time. The end time of the movement was the first point, after the time of peak velocity, when distance stopped increasing. MT was the difference between end and start times. We calculated the following additional trajectory variables: (1) average absolute acceleration $(|\bar{a}|)$ : average value, from movement start to end, of the rectified tangential acceleration (i.e., average of the absolute value of acceleration, or sum of the average acceleration and average deceleration); in Figure 2, $|\bar{a}|$ is the total (unsigned) area under the acceleration curve (acceleration and deceleration components; indicated by shading) divided by the MT; (2) peak acceleration: the first maximum of the acceleration time series after movement start; (3) peak jerk: the first maximum of the jerk time series after movement start; (4) integrated square jerk: the time integral, from movement start to end, of the square of the jerk time series; (5) path curvature: the "shortest possible path" of each movement was a straight line joining the start and end points of the movement; we defined curvature as the unsigned area between hand path and the shortest possible path, divided by the area of a circle with the shortest possible path as its diameter; and (6) velocity profile asymmetry: the contrast (difference divided by sum) between duration of acceleration phase (time of peak velocity minus time of movement start) and deceleration phase (time of movement end minus time of peak velocity).

Spatial accuracy. We measured movement extent as the distance from the hand's position at movement start to its position at movement end, and movement direction as the angle, counterclockwise from the $x$-axis, to the line containing the start and end positions of the movement. For each movement, we calculated extent error as the difference between movement and target extent, and directional error as the difference between movement and target direction. For each condition, we used each subject's mean values of these quantities as condition-level measures of systematic error. Because movement direction did not vary systematically across conditions in either group (see Results), we examined systematic extent error as a measure of constant error within a condition. Our primary measure of variable error was the square root of the generalized endpoint variance $\left(\sigma_{g}\right)$ (i.e., the square root of the determinant of the spatial covariance matrix $\mathbf{C}$ ). For a given set of movement endpoints, $\mathrm{C}$ is the matrix composed of $\left(S_{x x}, S_{x y}\right)$ (first row), and $\left(S_{y x}, S_{y y}\right)$ (second row), where $S_{x x}$ is the variance of the endpoints along the $x$-axis, $S_{y y}$ is the variance along the $y$-axis, and $S_{x y}\left(=S_{y x}\right)$ is the covariance between $x$ and $y$ positions. We used the square root of the determinant of $\mathbf{C}$ so we could express variable error in units of area. $\sigma_{g}$ subtracts the contribution attributable to any correlation between the $x$ and $y$ coordinates of the endpoints from the total endpoint scatter and thus measures variable error without redundancy. However, we also computed two additional measures of variable error, which are proportional to more commonly used measures of endpoint scatter. The total standard deviation $\left(S_{T}\right)$ is the vector sum of the standard deviation of the endpoints along $x\left(S_{x}\right)$ and along $y\left(S_{y}\right)$ [i.e., $\left(S_{x}{ }^{2}+S_{y}{ }^{2}\right)^{1 / 2}$ ]. To calculate the third measure of variable error, we first decomposed the vector from movement endpoint to target center into components parallel and perpendicular to the direction of the target and then calculated the interquartile range (difference between 75 th and 25th percentiles) along the parallel and perpendicular directions $\left(Q_{p a r}\right.$ and $Q_{\text {perp }}$, respectively). Our third measure of variable error was the total interquartile range $\left(Q_{T}\right)$, which computes the vector sum of parallel and perpendicular interquartile ranges: $Q_{T}=\left(Q_{p a r}{ }^{2}+\right.$ $\left.Q_{\text {perp }}{ }^{2}\right)^{1 / 2}$. Note that although, strictly speaking, "accuracy" refers to systematic error and "precision" refers to variable error, we follow the usage of accuracy common in the speed-accuracy literature, using the term for both systematic and variable error.

Timing. We recorded the movement onset latency as the time between the appearance of the target and the movement start time. Note that because of the task instructions ("move when ready"), this latency is not equivalent to a reaction time. We recorded the intertrial interval (ITI) as 


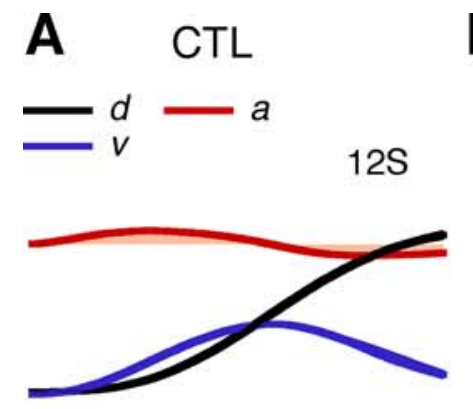

B

PD

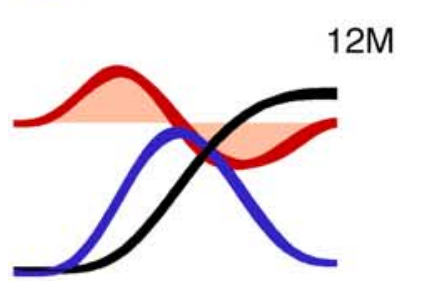

$12 \mathrm{~F}$
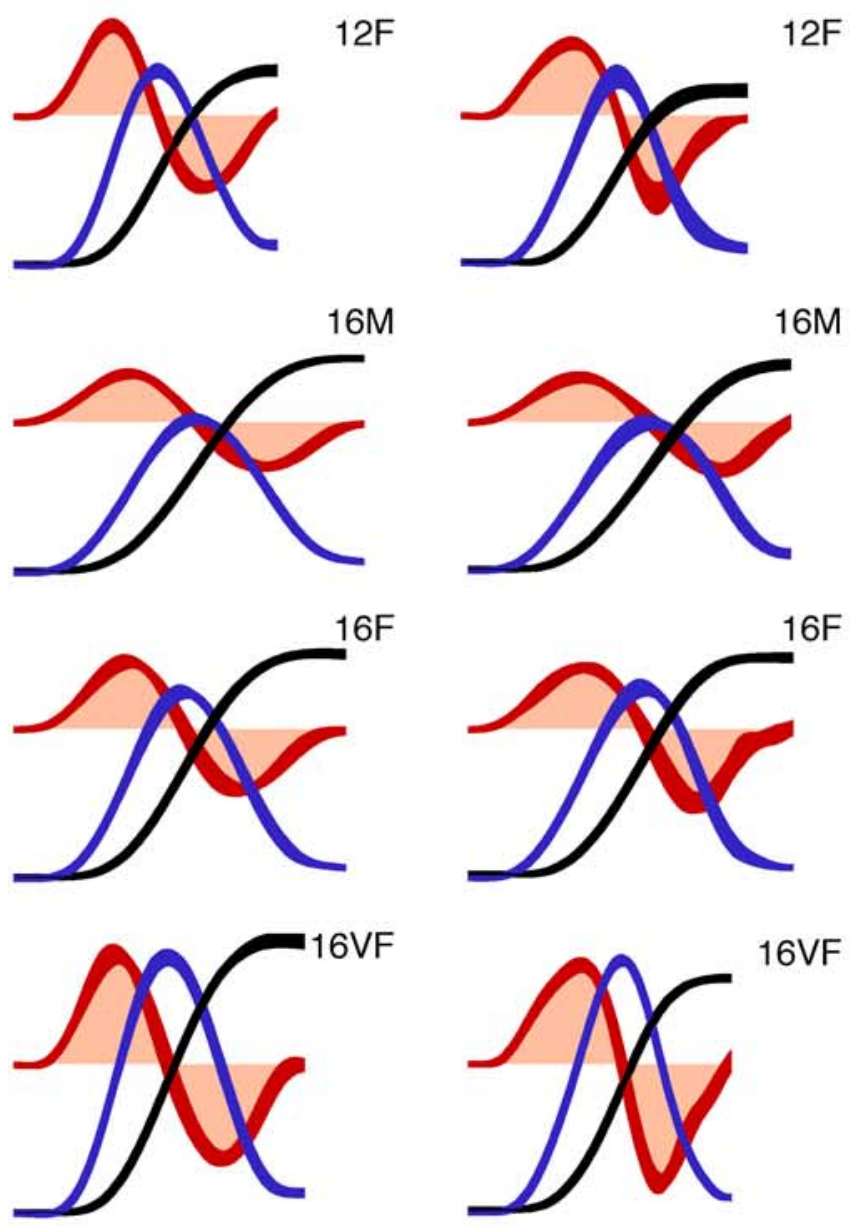

16VF

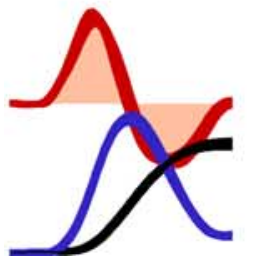

$6 \mathrm{M}$

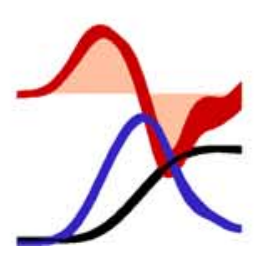

Figure 2. Movement kinematics for two representative subjects. $\boldsymbol{A}$, Time course of hand distance ( $d$, black), velocity ( $v$, blue), and acceleration ( $a$, red) for control (CTL) subject $C 3$ (see Table 1) in the seven task conditions. MT is indicated by the horizontal extent of the traces. Distance refers to the hand's distance from movement start point. Each trace is the mean time series for each kinematic variable across valid trials for each condition. Trace thick- the time between the end time of one movement and the start time of the next movement.

Energy measures. We calculated joint work and power using standard biomechanical equations (Hatze and Buys, 1977; Winter, 1990). The mass of the forearm and upper arm was calculated as a fraction of the subject's weight from published morphometric tables (Winter, 1990). We calculated joint angles and torques using previously described methods (Sainburg and Kalakanis, 2000).

Statistical tests. We used the JMP software package (version 6.0.3; SAS Institute, Cary, NC) to perform the following statistical tests: ANOVA, which always included group as a between-subject factor and condition as a repeated measure (within-subject factor); post hoc $t$ tests, corrected for multiple comparisons using Tukey's method of "honest significant difference"; simple linear regression; and linear models with standard least squares. Results were considered significant at $p \leq 0.05$.

Power analysis. We performed power analysis to assess the false negative rate for the effect of group. We designed our experiment to elicit marked differences in accuracy and trajectory measures across conditions. We decided a priori that the minimal meaningful difference (effect size) between groups should equal the average difference, for the variable of interest, between conditions. For example, if condition had a significant effect on variable error, then the effect size for group was the average difference in variable error across all conditions. Only negative results powered at 0.80 or above (at $\alpha=0.05$ ) were considered reportable.

\section{Results}

Subjects made self-paced planar arm movements aimed at visual targets without vision of their hand (Fig. 1A). On valid trials (peak velocity in the required range), they received visual feedback about their accuracy (Fig. 1B). No accuracy feedback was provided in void trials (Fig. 1C). After 20 valid trials, the required speed range and/or target distance changed to those for the next condition block. Figure 2 shows the average time course, across valid trials, of tangential acceleration, velocity, and distance from the origin for a representative control subject and for the most severely affected patient (i.e., the patient with the highest motor UPDRS score). The different combinations of target distance and peak velocity requirement had a systematic effect on all three of these variables, as well as on MT. In all conditions, movements consisted of smooth single movements, with symmetric bellshaped velocity profiles and single acceleration and deceleration peaks, indicating the absence of additional submovements. Moreover, the effect of condition on the velocity, acceleration, and displacement profiles was similar for controls and patients. This similarity indicates that our task design was able to elicit a single type of movement from both patient and control groups and allowed us to make subsequent comparisons.

We chose target distances and speed requirements that would elicit a speed-accuracy trade-off. Figure 3 illustrates this trade-off for two representative subjects. In the condition with a target distance of $12 \mathrm{~cm}$ and a slow speed requirement $(7-37 \mathrm{~cm} / \mathrm{s}$ ) (Fig. $3 A, B)$, movement endpoints for both the control subject and the patient clustered around the target, showing small systematic error (distance from center of target to average location of endpoints) and small variable error (spatial spread of endpoints). When the required speed was increased, both systematic and

$\leftarrow$

ness indicates the $95 \%$ confidence interval around the mean. Shading of the area under the acceleration trace indicates average velocity (area under acceleration curve). Traces are aligned to movement start time. Condition labels (12S, 12M, 12F; 16M, 16F, 16VF; 6M) indicate target distance $(6,12,16 \mathrm{~cm})$ and required speed range $(S, 17-37 \mathrm{~cm} / \mathrm{s} ; \mathrm{M}, 37-57 \mathrm{~cm} / \mathrm{s} ; \mathrm{F}, 57-77$ $\mathrm{cm} / \mathrm{s} ; \mathrm{VF}, 77-97 \mathrm{~cm} / \mathrm{s}$ ). Calibration (applies to all panels): horizontal, $200 \mathrm{~ms}$; vertical, $5 \mathrm{~cm}$ for d, $20 \mathrm{~cm} / \mathrm{s}$ for v, $330 \mathrm{~cm} / \mathrm{s}^{2}$ for a. $\boldsymbol{B}$, Same information as in $\boldsymbol{A}$, but for a single PD patient (subject P6) (see Table 1). 


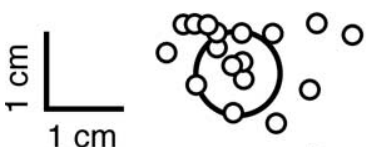

A 125

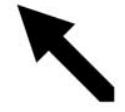

B 125

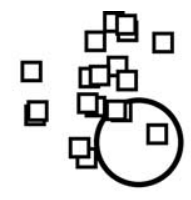

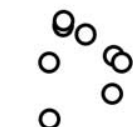
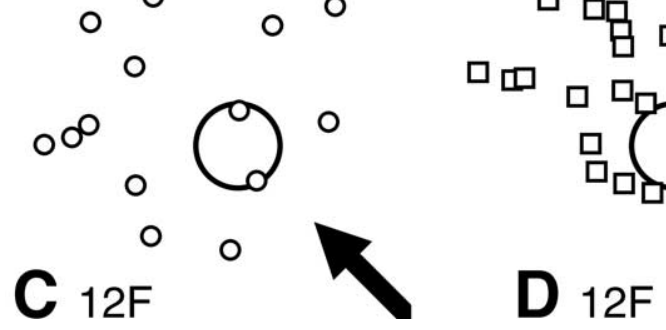

Figure 3. Illustration of speed-accuracy trade-off. $A$, Scatterplot of endpoints for valid trials of a single control subject (C6) (see Table 1) in condition $12 S$ (target distance, $12 \mathrm{~cm}$; required speed, $17-37 \mathrm{~cm} / \mathrm{s}$ ). Plotted is the hand's position ( $y$ vs $x$ ) at the end of each valid trial. The large circle indicates the target (only the smallest circle of the bull's eye is shown). The arrow shows the direction from the start circle (not shown) to the target, which approximately corresponds to the average movement direction. Calibration, $1 \mathrm{~cm}$. $\boldsymbol{B}$, Same data as in $\boldsymbol{A}$, but for condition $12 \mathrm{~F}$ (target distance, $12 \mathrm{~cm}$; required speed, $57-77 \mathrm{~cm} / \mathrm{s}$ ). $\boldsymbol{C}, \boldsymbol{D}$, Endpoint scatter for single PD patient (subject P5) (see Table 1) in conditions $12 S(\boldsymbol{C})$ and $12 F(\boldsymbol{D})$. Axis scaling is as in $\boldsymbol{A}$ and $\boldsymbol{B}$.

variable errors increased: the endpoints moved farther from the target and became more scattered (Fig. 3C,D).

\section{Speed and MT were successfully matched between groups}

We required subjects to make 20 valid movements in each condition to obtain movements of comparable speeds from control subjects and patients. Figure $4 A$ shows peak velocity, averaged across subjects in each condition, for valid trials in the control and PD groups. Peak velocity varied systematically across conditions and assumed values in four ranges, which corresponded to the imposed required speed ranges. The $\mathrm{S}$ and VF ranges yielded, respectively, the lowest and highest peak velocities, and the $M$ and $F$ ranges yielded peak velocities that did not vary with target distance. Notably, patients and control subjects achieved very similar peak velocities in each condition. Figure $4 B$ shows mean MT across conditions. As expected, conditions with higher peak velocity had lower MT values. As observed for peak velocity, MT was also very similar for patients and control subjects in all conditions. Although condition had a significant effect on peak velocity ( $p<0.0001$; ANOVA with condition as repeated measure, group as between-subject factor) and MT $(p<0.0001)$, there was no significant effect of group ( $p=0.49$ for peak velocity, 0.17 for $\mathrm{MT}$ ). The absence of a group effect indicates that the task design successfully yielded movements of equal speeds for the two subject groups. For details of statistical tests, see supplemental Table 2 (available at www.jneurosci.org as supplemental material).

\section{Patients showed normal speed-accuracy trade-off}

We next examined spatial accuracy for the valid trials. Figure 4, $C$ and $D$, shows mean values, in each group and condition, of systematic extent error and our primary measure of variable error $\left(\sigma_{g}\right)$. For a given target distance, both types of error increased as the required speed increased. The control and PD groups had similar values of systematic and variable error in each condition. The overshoot in Figure $4 C$ stems from the imposed difficulty in reaching the required velocity over the distance allowed by the target.

There was a significant effect of condition on both systematic and variable error ( $p<0.001$ in both cases), which demonstrates that the task indeed imposed a trade-off between speed and accuracy. However, there was no significant effect of group for either systematic ( $p=0.57)$ or variable $(p=0.26)$ error. Our additional measures of variable error yielded the same results: a significant effect of condition ( $p=0.03$ for $S_{T}$ and $Q_{T}$ ) but no effect of group ( $p=0.33$ for $S_{T}, 0.84$ for $Q_{T}$ ).

The effect size calculated from our a priori choice (i.e., average of the differences between conditions) was $0.96 \mathrm{~cm}$ for systematic error and $0.48 \mathrm{~cm}^{2}$ for variable error. Power for detecting such differences between groups was 0.81 and 0.98 , respectively. Thus, we achieved the desired control of the false negative rate.

If the speed-accuracy relationship is normal in PD, then it should be normal across all movement speeds and not just those in the required speed ranges. Therefore, we performed a comparison of peak velocity, MT, systematic extent error, and total variable error in the void trials. The results were the same as for the valid trials: these variables varied significantly across conditions but were not different between patients and control subjects. There was a statistically significant effect of condition but no effect of group on any of these variables (supplemental Table 2, available at www.jneurosci.org as supplemental material). Notably, patients did not move more slowly than control subjects in the void trials and maintained the same accuracy as control subjects even in trials for which they did not receive any accuracy feedback.

We examined the relationship between speed and accuracy in more detail by comparing the endpoint scatter of PD and control groups binned across narrow speed ranges for all movements (valid and void trials). Figure 5 shows that accuracy for the two groups was closely matched throughout the entire range of speeds. Note that, in this analysis, void trials were not confined to the lowest speed ranges; rather, valid and void trials from various conditions were mixed throughout all bins. Variable error (as measured by $\sigma_{g}$ ) was significantly affected by speed range ( $p=$ 0.03 ) but not by group ( $p=0.79$; ANOVA with speed range as repeated measure, group as factor). This result reinforces the finding that the relationship between speed and accuracy was normal in the PD group.

\section{Patients showed increased $N_{c}$}

Besides affecting endpoint accuracy, the various conditions affected another measure of performance, namely the number of trials needed to make 20 valid movements (trials to criterion, $N_{c}$ ). This measure varied systematically across conditions, revealing another aspect of task difficulty. Figure $6 \mathrm{~A}$ shows that, for subjects in both groups, $N_{c}$ was smallest when the target distance was $12 \mathrm{~cm}$ and the required peak velocity range was lowest (condition $12 \mathrm{~S})$. Subjects found this condition the easiest, in the sense that they reached 20 valid movements in little over 20 trials (i.e., there were very few void trials). However, with an increase in the peak velocity requirement, for a given target distance, all subjects generated more void trials and $N_{c}$ increased (Fig. $6 \mathrm{~A}$ ). Moreover, this increase in $N_{c}$ across conditions was greater for patients than for control subjects. There was a significant effect of condition $(p=$ 0.002 ) as well as group ( $p=0.02$; ANOVA with condition as 
repeated measure, group as factor) on $N_{c}$. Figure $6 B$ illustrates the effect of group on $N_{c}$ in a reliability analysis format. In this figure, a product-limit (Kaplan-Meier) curve indicates the proportion of subjects who reach criterion (across all conditions) versus movement number. A small fraction of subjects (15-20\%) reached criterion after 20 trials, which reflects the easiest condition (Fig. 6A). Then, as movement number increased, a greater proportion of subjects reached $N_{c}$ (Fig. $6 B$, $12 S)$. The curve for the PD group, however, increased significantly more slowly, as evident in Figure $6 B$ and reflected in the log-rank statistic $\left(\chi^{2}=7.00 ; p=0.0082\right)$. Critically, the difference in $N_{c}$ between groups contrasted with the difficulty effect of the speed-accuracy trade-off, which did not differ between patients and controls. This dissociation is not compatible with a compensatory explanation for bradykinesia: patients were as accurate as controls yet still required more trials to make 20 valid movements.

A greater number of trials required to make a fixed number of valid movements implies a greater number of void trials. We investigated the nature of the void trials by examining the distributions of peak velocity in all trials (valid and void) (Fig. 7). These distributions are shown as histograms in Figure $7 A$ and as probability density functions in Figure $7 B$. Each panel in Figure 7 shows data for a particular condition, and vertical dashed lines indicate the required speed range for each condition. Because we required every subject to make 20 valid movements in each condition, the area under the traces between the vertical dashed lines (i.e., the total number of valid trials) had to be the same for patients and control subjects. Any difference in $N_{c}$ therefore had to be reflected in differences outside the dashed lines (void trials). In some conditions, such as $12 \mathrm{~S}$ (Fig. $7 \mathrm{~A}$, top left), the majority of peak velocities fell within the required range, which indicates a low number of void trials and corresponds to low values of $N_{c}$. In other conditions, such as $12 \mathrm{~F}$ (Fig. $7 A$, bottom left), there were peak velocities both inside and outside the required range, indicating the presence of void trials (velocities lower and higher than the required range) besides valid ones.

Two features are immediately apparent in the histograms in Figure $7 A$ : in conditions requiring higher speed for a given target distance, the peak velocities of valid trials migrate toward the left edge of the required range, and the number of void trials increases. This was true for patients and control subjects and is consistent with (although on its own does not establish the presence of) the speed-accuracy trade-off of the task: in conditions in which spatial accuracy deteriorated (Fig. 4C,D), subjects' speeds concentrated around the minimum required speed, which led to more movements with speeds just below it. A third notable feature of the histograms is that the speeds of the patients' void trials are in the same range as those for controls subjects' void trials: the patients make more void movements but appear to select these movements from a distribution similar to that of control subjects.
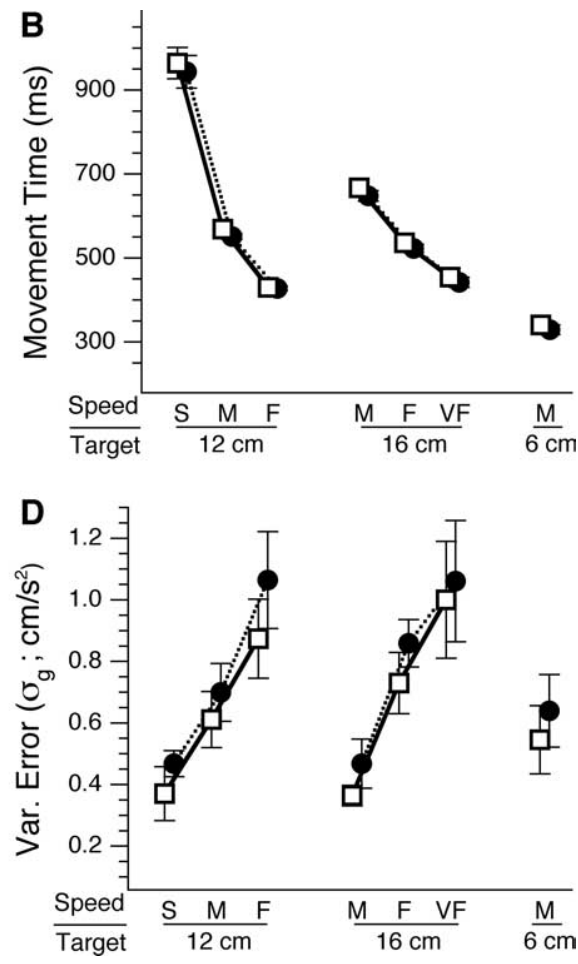

Figure 4. Speed-accuracy trade-off performance. Each panel shows values (means, across subjects, of subject's mean values, subject's mean value for valid trials in each condition. Error bars indicate SE. Note that Speed near the horizontal axis in all panels refers to range of required speeds, whereas peak velocity on the vertical axis in $A$ refers to the speed actually attained by subjects. Where error bars are not visible, they are smaller than the plot symbol indicating the mean (e.g., in $\boldsymbol{A}$ and $\boldsymbol{B}$ ).

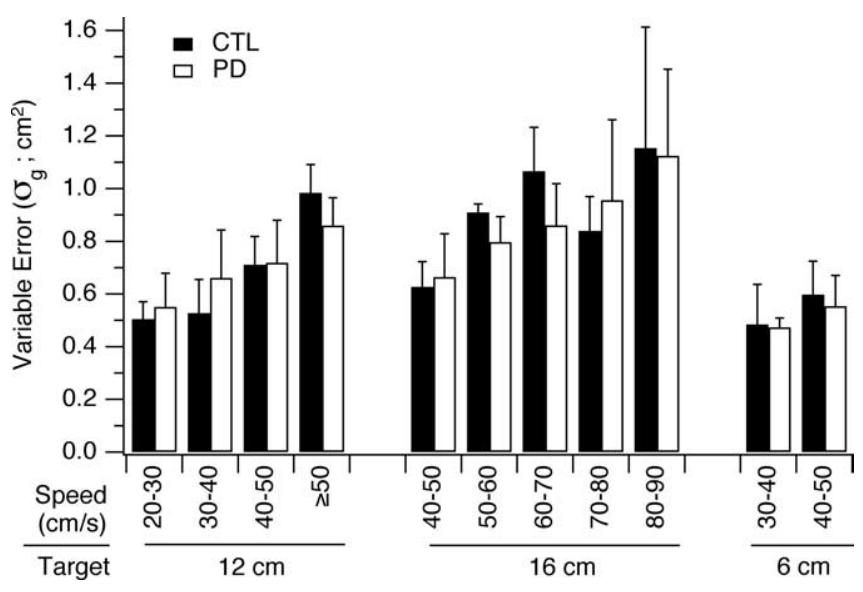

Figure 5. Comparison of variable error between PD and control (CTL) groups, across the entire range of speeds for all trials. The range of recorded peak velocities for movements to a given target $(6,12$, and $16 \mathrm{~cm})$ is divided into bins of $10 \mathrm{~cm} / \mathrm{s}$ width, and all subjects' variable error $\left(\sigma_{g}\right)$ is averaged within each bin according to the movements' peak velocities, regardless of whether the trial was valid or void. Filled bars, Control group; open bars, PD group. Error bars indicate SE.

There was no significant difference between groups in the mean, variance, or skewness of the peak velocity distributions $(p<$ 0.05) (supplemental Table 2, available at www.jneurosci.org as supplemental material). This was true whether all trials were con- 

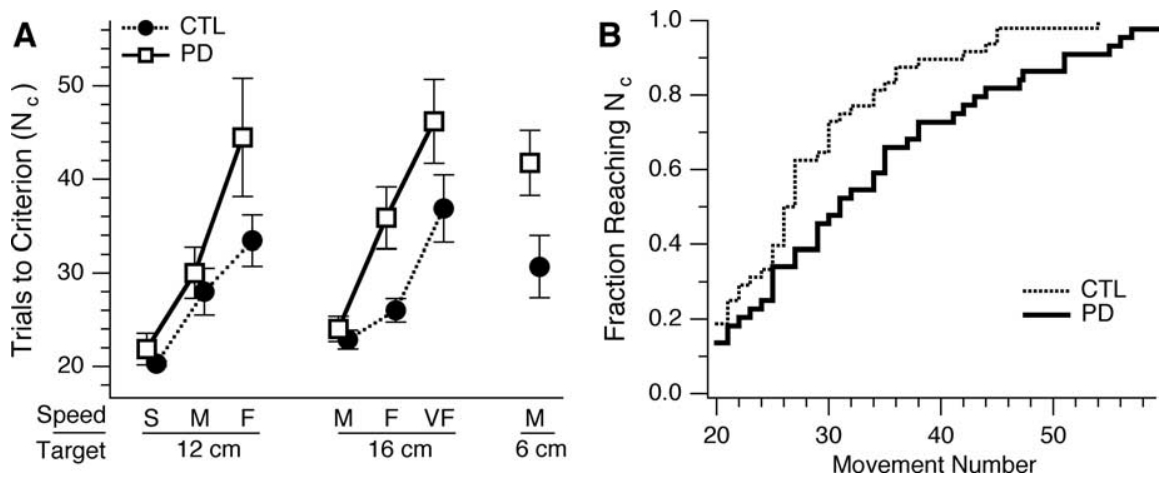

Figure 6. Trials to criterion $\left(N_{c}\right) . \boldsymbol{A}$, Number of trials needed by subjects to make 20 movements in required speed range of each condition. Plotted values are means, across subjects in each group, of every subject's $N_{c}$ value for each condition \pm SE. Symbols and the $x$-axis are as in Figure 4. B, Product-limit (Kaplan-Meier) reliability plot of $N_{c}$ for the subject groups. The $x$-axis indicates the number of trials, and the $y$-axis shows the proportion of subjects reaching criterion ( 20 valid trials) in each group. All conditions are included, so that each step reflects, at a minimum, one subject reaching criterion in a single condition. CTL, Control.

sidered or only void ones to the left of the minimum required speed.

Analysis of the raw distributions in Figure $7 A$ thus suggests that the greater number of void trials in patients was not attributable to a systematic shift to lower speeds but rather to an increase in the proportion of slower movements selected from the same speed values available to control subjects. We tested this hypothesis through analysis of the probability density functions of the speed distributions, calculated with nonparametric density estimation (Silverman, 1986). This method yields estimates of the underlying probability functions from which movement speed is selected (Fig. 7B). To permit comparison of values between subject groups, we did not normalize these functions to the total number of trials for each group: the area under each trace in Figure $7 B$ reflects the total number of trials for each group in each condition. The shaded region between the two traces in each panel indicates the additional void movements made by patients compared with control subjects. A striking feature of the traces in Figure $7 B$ is that the patient's probability density functions differ from those of control subjects much more in amplitude than width: the patients' traces appear to be vertically scaled versions of the controls' traces, rather than broader or horizontally shifted. We quantified these features by fitting each trace to a Gaussian function with amplitude $K$, mean $v_{0}$, and SD $\sigma_{v}$ through least-square curve fitting. The resulting values for these fit parameters are shown in Figure 8 . The change in $K$ (Fig. $8 \mathrm{~A}$ ) paralleled the change in $N_{c}$ (Fig. $6 A$ ) across subjects and conditions: patients' values of $K$ diverged from those of control subjects in the same conditions as for $N_{c}$. The mean $\left(v_{0}\right)$ and width $\left(\sigma_{v}\right)$ of the Gaussians, in contrast, showed no such divergence (Fig. $8 B, C$ ). This analysis revealed two important features of speed selection mechanisms in the two subject groups: (1) to generate the required number of movements in the imposed speed range, subjects in both groups selected movement speeds from a single underlying probability distribution; and (2) patients selected speeds from a probability distribution that is essentially an amplified version of the one that governs normal speed selection. This suggests that patients have access to the same speed selection mechanisms as control subjects and emphasizes the probabilistic nature of bradykinesia: it consists of a higher probability of making slower movements despite preserved motor execution at higher speeds.

\section{Patients' increased $N_{c}$ reflected reduced} movement vigor

Why was $N_{c}$ higher for some conditions than others? The two features that distinguished conditions were target distance and required peak velocity. An increase in speed for a given target distance, coupled with a reduction in target distance, requires a larger and quicker acceleration and deceleration. This is captured by the average absolute acceleration $(|\bar{a}|)$ (i.e., the time average, from movement start to end, of the absolute value of the tangential acceleration time series). This measure corresponds to the shaded regions of the acceleration traces in Figure 2 divided by MT and has a positive contribution from both acceleration and deceleration. Thus, we chose $|\bar{a}|$ as a single continuous movement variable that varied parametrically across conditions and asked how much this variable accounted for the variation in $N_{c}$. Figure 9 is a plot of $N_{c}$ versus $|\bar{a}|$ for patients and control subjects. There is a clear linear relationship between $N_{c}$ and $|\bar{a}|$ for both groups, with $N_{c}$ increasing as $|\bar{a}|$ increases. However, there is a different dependence of $N_{c}$ on $|\bar{a}|$ for patients and control subjects: $N_{c}$ increases more rapidly, as $|\bar{a}|$ increases, for patients. To quantify these relationships and measure differences between subject groups, we modeled $N_{c}$ as a function of $|\bar{a}|$ and group (linear model, tested using standard least squares) (Sokal and Rohlf, 1981). We found a significant linear component for the overall model $(p<0.0001)$, which accounted for $91 \%$ of the variance (i.e., Pearson correlation coefficient, or $\left.R^{2}=0.91\right)$. There were significant linear contributions of $|\bar{a}|$ to $N_{c}$ in the individual subject groups $(p<0.0001$ for control and PD), with $R^{2}$ values of 0.88 for the control group and 0.89 for the PD group. There was also a significant effect of group $(p<0.0001)$. More importantly, there was a significant interaction between group and $|\bar{a}|$ in the model $(p=0.02)$. A significant interaction between group and $|\bar{a}|$ in their contribution to $N_{c}$ establishes that the group-specific linear relationships between $N_{c}$ and $|\bar{a}|$ had significantly different slopes. In other words, as evident in Figure 9, $N_{c}$ had a steeper dependence on $|\bar{a}|$ in patients than in control subjects. We refer to the slope between $N_{c}$ and $|\bar{a}|$ as $S_{N}$.

The strong linear relationship between $N_{c}$ and $|\bar{a}|$ supports the hypothesis that average absolute acceleration, as a single parameter, captures a large amount of task difficulty, measured as the number of trials needed to reach criterion. This relationship alone, however, may reflect contributions from both the speedaccuracy trade-off and movement energetic costs. Accuracy was indeed related to $|\bar{a}|$, which supports the idea that the speedaccuracy trade-off influences $N_{c}$. Specifically, a linear model of accuracy (expressed either as systematic or variable error) as a function of $|\bar{a}|$ and group yielded a significant contribution of $|\bar{a}|$ (for systematic extent error: $p=0.0002, R^{2}=0.84$; for total variable error: $\left.p=0.0065, R^{2}=0.69\right)$. However, there was no significant effect of group ( $p=0.44$ for systematic error; $p=0.91$ for variable error), and no significant interaction between $|\bar{a}|$ and group ( $p=0.93$ for systematic error; $p=0.30$ for variable error). Crucially, therefore, the slope of the relationship between accuracy and $|\bar{a}|$ was the same for both groups. This is consistent with the finding that there was no difference between the two groups for systematic or variable error across conditions (Fig. 4C,D). 

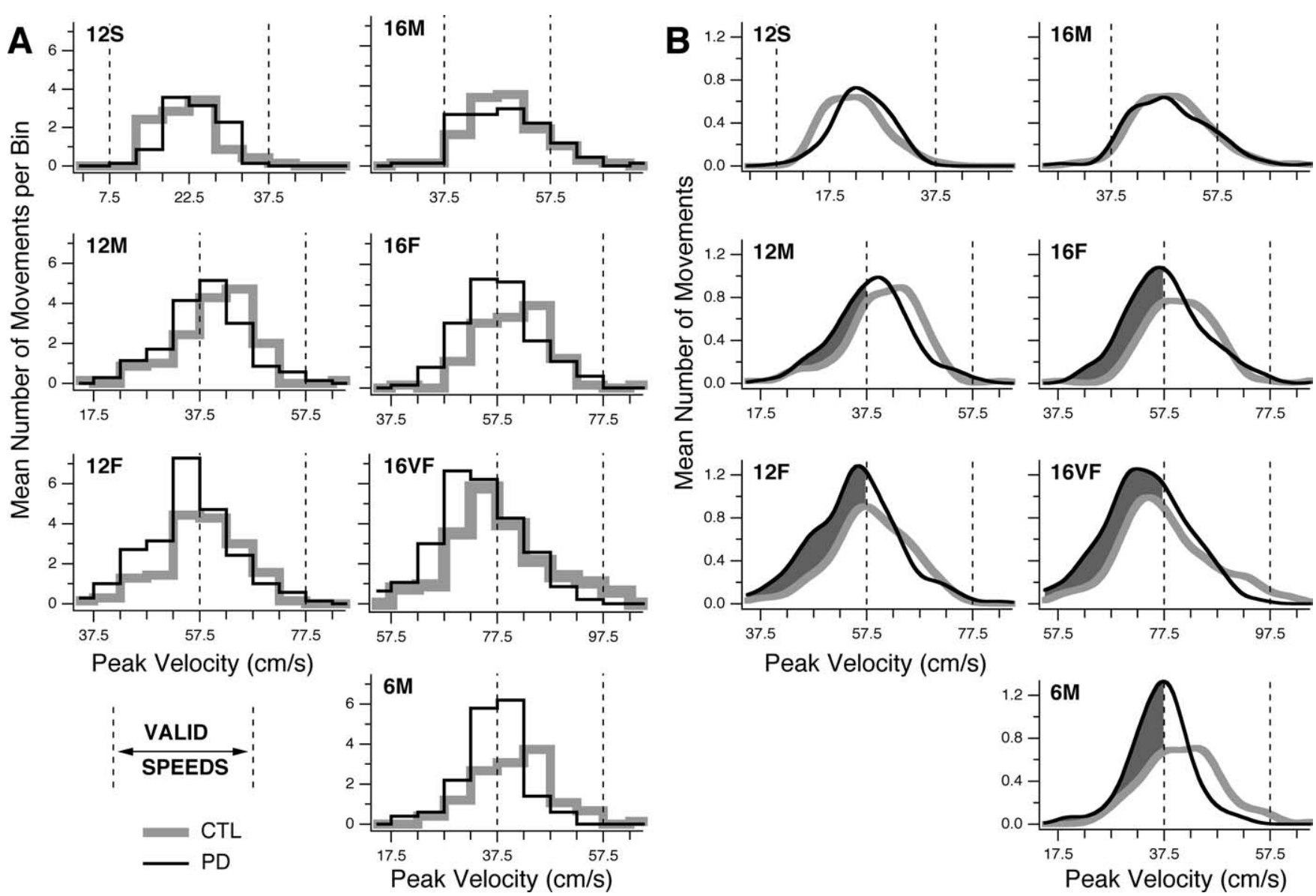

Figure 7. Distribution of peak velocity in all trials (valid and void) for each condition. Labels for the conditions are as in Figure 2. A, Histogram representation of peak velocity distributions. Each panel shows, for a single condition, the number of movements, averaged across subjects in each group, with peak velocity in the range defined by each bin (bin width, $5 \mathrm{~cm} / \mathrm{s}$ ). Thin black lines indicate data from patients, and thick gray lines indicate data from control subjects. Dashed vertical lines mark the range of required speeds for each condition: movements within these lines are valid trials; those outside are void trials. Note that the scale of the horizontal axes is the same across all panels; differences in range are attributable only to horizontal shifts. The scale of the vertical axes is the same for all panels. $\boldsymbol{B}$, Nonparametric probability density estimation of peak velocity distributions, based on same data as in $\boldsymbol{A}$. Each trace is the sum of normalized Gaussian functions (SD, $2.5 \mathrm{~cm} / \mathrm{s}$ ), with each movement contributing a Gaussian function (kernel) centered on its peak velocity (Silverman, 1986). This sum is an estimate of the probability density function underlying movement speed distributions. The vertical axis indicates the number of movements, averaged across subjects in each group, with peak velocity within $1 \mathrm{~cm}$ of the corresponding $x$-axis value. Note that the functions are not normalized, to allow comparison of distributions between subject groups and across conditions. Shading indicates the difference between patients' and control subjects' distributions for void trials. Trace thickness, trace shading, and horizontal axes are as in A. CTL, Control.

The results of this analysis are thus that $N_{c}$ depends linearly on $|\bar{a}|$ and that this dependence is steeper (higher $S_{N}$ ) for patients than for control subjects. Moreover, accuracy also depends linearly on $|\bar{a}|$, but this dependence has the same slope in the PD and control groups. The finding of a significant interaction effect between group and $|\bar{a}|$ on $N_{c}$ means that there was a different effect of $|\bar{a}|$ on $N_{c}$ for the two groups. On the other hand, the lack of difference, between groups, in the relationship between accuracy and $|\bar{a}|$ implies that the speed-accuracy contribution to difficulty was the same for the two groups. The dependence of $N_{c}$ on $|\bar{a}|$ for both groups means that, as conditions became more difficult, subjects made more void trials between valid ones. Because the speed-accuracy contribution to difficulty was the same for the two groups, there must be another explanation for the greater number of void trials per successful ones in the PD group. Combined, these results point to the existence of a second component of task difficulty, which is independent of the speed-accuracy trade-off. We propose that $|\bar{a}|$ also represents a movement energy cost, to which the PD patients were more sensitive.

Average absolute acceleration measures how rapidly a movement must start and stop. When multiplied by the mass of a subject's arm, $|\bar{a}|$ is a measure of rate of change of momentum and is closely related to the rate of energy expenditure (average power). We found that the values of $|\bar{a}|$ obtained in our study closely correlated with the square root of the power expenditure for the entire arm (total muscle power at the elbow and shoulder joints, calculated from joint velocity, joint acceleration, and inertia of arm segments), averaged over movement duration $\left(R^{2}=\right.$ $0.76 ; p<0.001)$. Compared with $|\bar{a}|$, the square root of the average arm power accounted for a slightly smaller amount of the variance in $N_{c}$ across conditions $\left(R^{2}=0.86\right.$ for control and 0.73 for PD vs 0.87 and 0.89 , respectively, for $|\bar{a}|)$. Thus, $|\bar{a}|$ is highly correlated with movement energy cost. We therefore conclude that the increased slope $\left(S_{N}\right)$ of the $N_{c}$ versus $|\bar{a}|$ relationship in the PD group indicates exaggerated sensitivity to movement energy cost.

If the increase in $S_{N}$ is specifically caused by PD, we would expect this slope to be influenced by disease severity. We calculated individual values of $S_{N}$ for each PD patient and plotted these values against each patient's motor UPDRS score (Fig. 10). The result was a significant linear component $(p=0.004)$, with the clinical score accounting for $86 \%$ of the variance of the slope. 


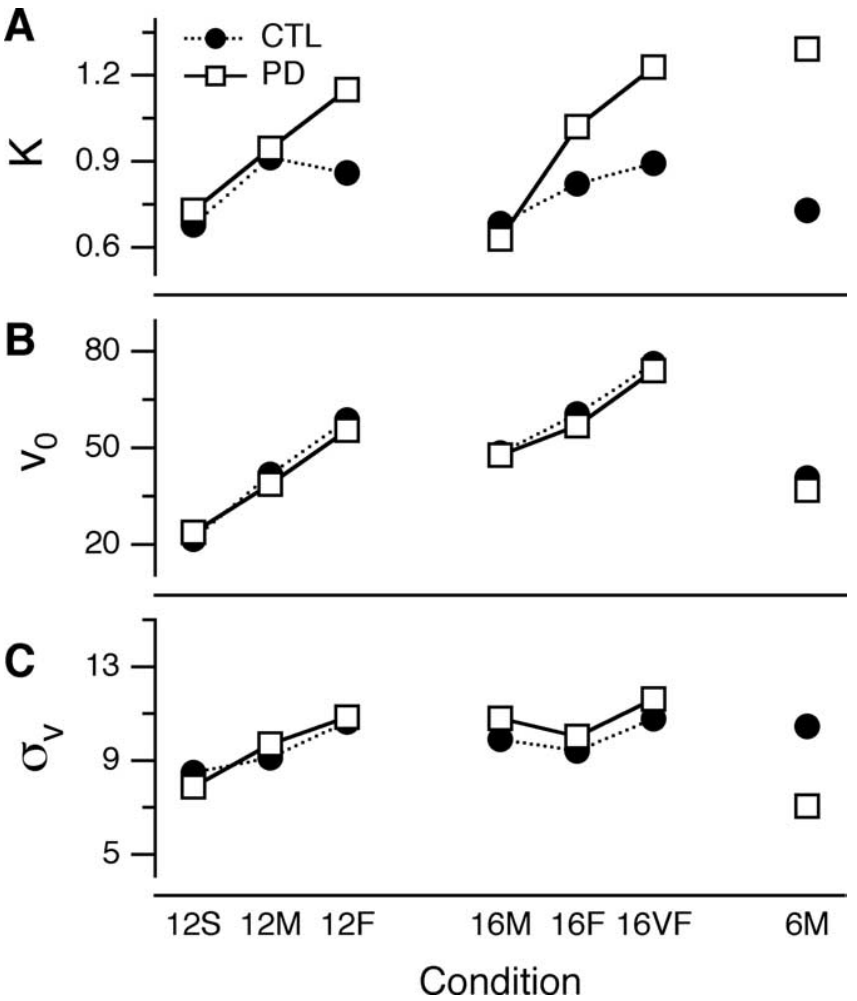

Figure 8. Parameters of Gaussian curve fits to the probability density functions for peak velocity in Figure $7 B$. Each trace in Figure $7 B$ was fitted, through least-squares methods, to a Gaussian function (i.e., $y=K e^{-\alpha}$, where $\alpha=\left[\left(v-v_{0}\right) / \sigma_{v}\right]^{2}, K$ is the peak amplitude, $v_{0}$ is the mean, and $\sigma_{v}$ is the SD. The Gaussian's parameters $K$ (peak), $v_{0}$ (mean), and $\sigma_{v}$ (SD) are shown, respectively, in $\boldsymbol{A}-\boldsymbol{C}$ versus the experimental condition. SDs of the estimates of the parameters are smaller than the height of the symbols of the plot for all values shown in the plot, and therefore are not visible. Labels for the conditions are as in Figure 2. Open squares and solid lines refer to data from PD patients; filled circles and dotted lines refer to data from control (CTL) subjects.

This result further indicates that the slope, $S_{N}$, is a biologically meaningful measure affected by PD.

Note that a reduction in movement vigor is not equivalent to generalized apathy, or global lack of energy, such as can be caused by affective disorders. If this were the case, patients should have shown reduced movement vigor (higher $N_{c}$ ) in all conditions, regardless of movement kinematics. Instead, patients' movement vigor was closely related to average absolute acceleration, pointing to an impairment in a very specific (motor) type of motivation, rather than a general lack of enthusiasm.

\section{Possible confounds}

A possible objection to our interpretation of the increase in $S_{N}$ is that although PD patients did not show any degradation of accuracy with increasing difficulty, they might exhibit degradation of other trajectory variables, such as curvature and integrated square jerk, known to be optimized quantities in current computational models (Todorov, 2004). We compared additional kinematic features of valid movements across conditions and groups. There was a significant effect of condition $(p<0.05)$ (see supplemental Table 3, available at www.jneurosci.org as supplemental material, for statistical test details) but no significant effect of group ( $p>0.05$ ) for the following kinematic variables: average absolute acceleration $(|\bar{a}|)$, integrated squared jerk, path curvature, peak acceleration, peak deceleration, peak jerk, time of peak acceleration, time of peak deceleration. Additionally, there was

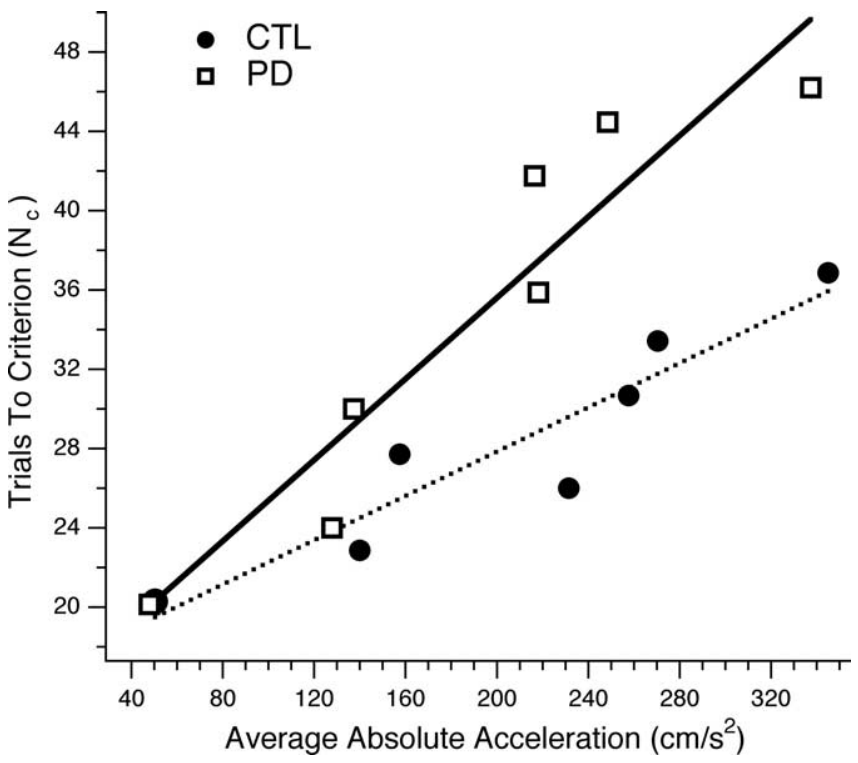

Figure 9. Relationship between trials to criterion $\left(N_{c}\right)$ and average absolute acceleration $(|\bar{a}|)$. Each $(x, y)$ point represents the mean, across subjects in each group, of every subject's $N_{c}$ and average absolute acceleration $(|\bar{a}|)$ for each condition. Lines indicate linear regression fits for $N_{c}$ versus $|\bar{a}|$. Open squares and the solid line indicate patients; filled circles and the dotted line indicate control (CTL) subjects. The linear fit for control group (dotted line) was as follows: $p<0.0001 ; R^{2}=0.88$; slope, 0.05 trials $/ \mathrm{cm}^{2} / \mathrm{s}^{2}$; intercept, 16 trials. The linear fit for the PD group (solid line) was as follows: $p<0.0001 ; R^{2}=0.89 ;$ slope, 0.10 trials $/\left(\mathrm{cm} / \mathrm{s}^{2}\right)$; intercept, 15 trials.

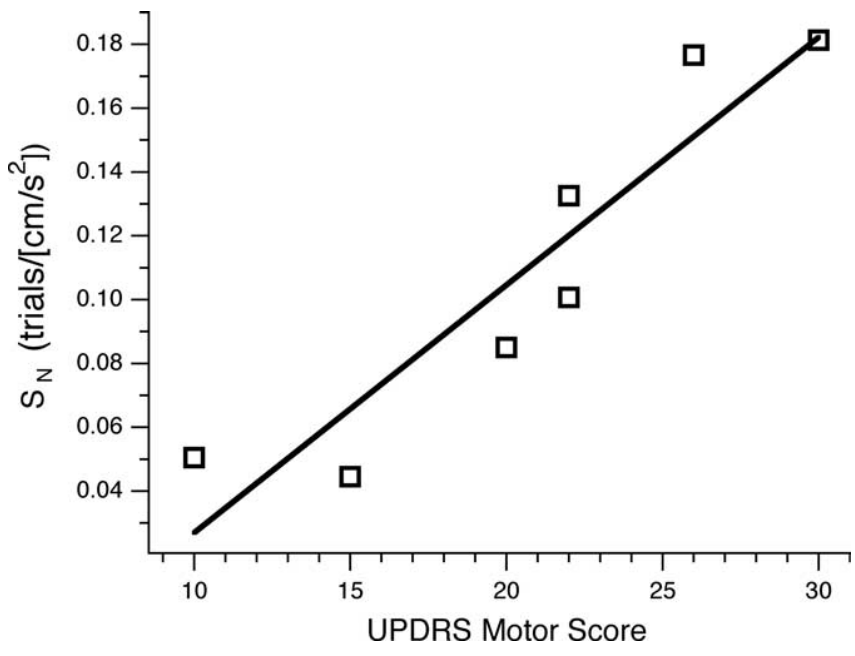

Figure 10. Correlation between sensitivity of movement speed selection to movement energy requirements and clinical status. The graph shows the relationship between the slope of the $N_{c}$ versus $|\vec{a}|$ line fit $\left(S_{N}\right)$ for each patient and the patient's motor UPDRS score. The linear fit (solid line) was as follows: $p<0.0044 ; R^{2}=0.86$; slope, $7.8 \times 10^{-3}\left[\right.$ trials/(cm/s $\left.\left./ \mathrm{s}^{2}\right)\right] /($ UPDRS point); intercept, $-50 \times 10^{-3}$ trials $/\left(\mathrm{cm} / \mathrm{s}^{2}\right)$.

no significant effect of condition or group on movement direction or velocity profile asymmetry. Therefore, the change in $S_{N}$ cannot be attributed to any execution-related variable. It should be emphasized that this was true for $|\bar{a}|$ itself, indicating that patients had no trouble executing movements of high $|\bar{a}|$; they were just less likely to do so.

Another possible objection is that learning or fatigue might have contributed to task performance, with each differentially affected by PD. If either learning or fatigue had an effect, then the proportion of void trials would have been different earlier in each 
condition block compared with later: a learning effect would produce more void trials earlier in a block; fatigue would, conversely, lead to increasingly slower speeds, and thus a higher frequency of void trials, in later portions of the block. We addressed this by comparing the number of trials needed to reach 10 valid movements $\left(N_{10}\right)$ to $N_{c}$. If learning and fatigue effects were absent (or perfectly balanced), it should take twice as many trials to reach $N_{c}$ (i.e., 20 valid movements) compared with $N_{10}$. We performed a linear regression of $N_{c}$ versus $N_{10}$, expecting a slope $>2$ if learning occurred and $<2$ if fatigue had an effect. We found that this relationship was strongly linear for both groups $(p<0.0001)$, accounting for $88 \%$ of the variance in the control group and for $77 \%$ in the PD group. The slopes for the control and PD groups (respectively, 1.8 and 1.7) were slightly smaller than 2, indicating that all subjects needed fewer trials between the 10th and 20th valid movements than for the first 10 . This is consistent with a small learning effect. Notably, these slopes were not significantly different for the two groups ( $p=0.83$; linear model for $N_{c}$ as function of group and $N_{10}$ ), indicating that the contributions of learning and fatigue to performance were not different between the two groups. Thus, the difference in $N_{c}$ between control and PD groups cannot be explained by a greater susceptibility of the PD group to learning or fatigue effects.

A third alternative explanation is that PD patients may have been less sensitive to velocity error (VE). After each trial, subjects received velocity feedback. Specifically, after a void trial, they had to estimate how much faster they needed to move on the next trial to reach the required speed. If PD affected the ability to process $\mathrm{VE}$ information, or if it blunted the perceived value of $\mathrm{VE}$, then PD subjects might make a smaller speed increase than control subjects after a void trial. This would increase the chance that the next trial might also be void and thus lead to a higher proportion of slower movements. We defined VE as the difference between the peak velocity of a void trial and the minimum required peak velocity for each condition. We then calculated the change in peak velocity after each void trial $(\Delta V)$ and the slope resulting from linear regression of $\Delta V$ against $V E$ for each subject. This slope was $0.52 \pm 0.04$ (mean $\pm \mathrm{SE}$ ) for the control group and $0.63 \pm 0.05$ for the PD group. These values are not significantly different ( $p=0.14, t$ test), with the mean value for patients showing a trend toward a greater, not smaller, value than for control subjects. Thus, patients did not make smaller speed increases after void trials.

Conversely, the PD group may have been inordinately sensitive to spatial error. After each valid trial, subjects received visual feedback about their spatial performance, in the form of the white square indicating their endpoint and the number of "smiley" faces. A common reaction to a large spatial error was to slow down on the next movement. If PD impaired processing of this spatial error, whether through impairment of visuospatial function or through a distortion of a value system attaching importance to a given spatial error, then patients might have slowed down more than control subjects after each valid trial, increasing the chance that the next trial would be void. We calculated $\Delta V$ as the change in peak velocity from after each valid trial (i.e., those trials in which subjects received spatial feedback). For each subject, we then calculated the slope of the relationship between $\Delta V$ and spatial error. We used extent error as a measure of spatial error, because it was much more sensitive to velocity than directional error. This slope was $-1.6 \pm 0.5$ (mean $\pm \mathrm{SE}$ ) for the control group and $-2.0 \pm 0.7$ for the PD group, with no significant difference ( $p=0.21, t$ test). Thus, patients did not excessively reduce their speed, after valid trials, because of increased sensitivity to spatial error.

We also addressed whether our results could be attributable to a co-contraction strategy, perhaps accentuating rigidity. It is plausible that, if movement variability were increased in PD, cocontraction could arise as a compensatory response and reduce variability by increasing the arm's mechanical impedance. Cocontraction would then impose an energy cost, manifested as reluctance to generate large average acceleration. As noted above, however, there was no effect of fatigue in the PD patients, which makes co-contraction an unlikely explanation for our results. Additionally, there was no correlation between $S_{N}$ and rigidity scores for the dominant arm $\left(p=0.77 ; R^{2}=0.02\right.$; simple linear regression). Thus, the contribution of rigidity to limb impedance also did not seem to be a factor.

Finally, we considered the possibility that differences in movement preparation times might contribute to differences in $S_{N}$. Our task did not assay reaction time (defined as the shortest achievable interval between a "go" signal and a response): subjects were instructed to move only when ready, and trials were performed in blocks, with the same target and speed requirements within each block. The ITI, however, reflects movement preparation time, because subjects could start planning the next movement as soon as the current movement ended. Movement onset latency (the interval between target appearance and movement start) could potentially be longer than the underlying reaction time, suggesting that the movement is still under preparation when the target appears; or it could be as short as the reaction time, suggesting that the movement is fully specified when the target appears. ITI was significantly affected by condition $(p<$ $0.001)$ and group $(p=0.04)$, with higher values in PD patients $(5890 \pm 150 \mathrm{~ms}$; mean $\pm \mathrm{SE})$ than controls $(5440 \pm 90 \mathrm{~ms})$ and a significant interaction between condition and group $(p=$ $0.004)$. Onset latencies, on the other hand, were not significantly affected by condition $(p=0.08)$ or group $(p=0.69)$ and were $150 \pm 30$ for controls and $180 \pm 40$ for patients.

We also modeled ITI as a function of $|\bar{a}|$ and group (linear model, tested using standard least squares) and found a significant effect of group $(p=0.02)$, a significant effect of $|\bar{a}|(p=$ $0.0001)$ and no interaction $(p=0.89)$. Notably, ITI was inversely correlated with $|\bar{a}|$ : subjects shortened their ITI in conditions requiring higher average acceleration. There was no significant relationship between movement onset latency and $|\bar{a}|$.

The results for ITI and onset latency show that PD patients used more time than controls to prepare movements, regardless of condition. Because movement onset latencies were shorter than published simple reaction times for pointing movements (Jeannerod, 1986), movement plans were likely fully specified at the time of target appearance; specifically, the similarity of latencies between patients and controls suggests that our task design succeeded in implementing, for both groups, a "go-when-ready" protocol, rather than a reaction-time one. Patients were thus not rushed to start their movements. The inverse correlation between ITI and $|\bar{a}|$ makes it unlikely that difficulties with movement preparation underlie the speed-accuracy trade-off or the relationship between $N_{c}$ and $|\bar{a}|$ : in conditions of greater difficulty and spatial error, all subjects shortened their ITI, while keeping the onset latency unchanged, rather than increasing either ITI or onset latency. This result further argues against apathy or general lack of motivation as the cause of higher $N_{c}$ values in patients. 


\section{Discussion}

Bradykinesia in early PD provided the opportunity to test two alternative hypotheses about MT selection. The first hypothesis was that bradykinesia is attributable to an impaired speed-accuracy trade-off or, more generally, to impaired trajectory optimization (compensatory bradykinesia). The second hypothesis was that patients show a reluctance to make fast movements because of the energy expenditure required, although they are capable of executing them as well as control subjects and without expending more energy. This decreased movement vigor would be reflected in an increased probability of patients selecting a slow movement speed on any given trial, which would lead to an increased $N_{c}$. We found that patients were able to move as fast as age-matched controls without any compromise in endpoint accuracy, trajectory quality, or energy expenditure. Both patients and controls showed an increase in $N_{c}$ as a function of task difficulty and energy requirement, expressed by the average absolute acceleration $(|\bar{a}|)$. However, patients showed an increased sensitivity to condition difficulty, with a larger increase in $N_{c}$ for a given increase in $|\bar{a}|$ compared with controls, despite normal movement trajectories and preserved endpoint accuracy, which indicates that patients are more likely than controls to move slowly when the energetic demands of a movement task increase.

Our task revealed a feature of bradykinesia that has been implicit in clinical descriptions but had not been quantitatively demonstrated, namely, that it is a probabilistic phenomenon. PD patients in our study moved more slowly than normal although their ability to move normally was fully preserved. The higher frequency of slower movements generated by patients, coupled with the similarity of speed distributions between patients and control groups, indicates a "choice" (albeit implicit) of slower movements rather than an inability to execute fast accurate ones. Our results establish that this speed selection abnormality was attributable to increased sensitivity to the energetic demands of the movements and not to a change in the speed-accuracy trade-off.

Although the energetic cost of a movement has been introduced as an optimization term in a recent computational model of motor control (Todorov and Jordan, 2002), its contribution to motor planning had not been experimentally demonstrated. A major obstacle to such a demonstration is that the speed-accuracy trade-off and energy cost usually covary in normal movements and thus would be difficult to disambiguate: slowing down could always be explained as the result of more stringent accuracy requirements (Tanaka et al., 2006). We identified a measure of bradykinesia (slope of $N_{c}$ vs $|\bar{a}|$, or $S_{N}$ ) with an abnormal relationship to movement energy but a normal relationship to the speedaccuracy trade-off, which allowed us to show that energy cost is an independent determinant of movement speed.

An explanation offered for bradykinesia is that it is a compensatory response to increased endpoint variability, that is to say, compensation for a primary impairment in the speed-accuracy trade-off (Sheridan and Flowers, 1990; Phillips et al., 1994). We envisioned a second mechanism, supported by our results: for a given task, there is a shift in the balance between the perceived reward of arriving to the target quickly and the amount of effort or energy required to achieve the fast movement. The framework of cost and reward has garnered a great deal of recent experimental and theoretical attention and is directly related to the role that has been proposed for dopamine in the modulation of motivational state (Schultz, 2006). Interestingly, although PD is a dopamine deficiency disease, its principal clinical signs have not gen- erally been considered in terms of cost and reward. Perhaps this is because PD primarily affects movement parameters, which are specified implicitly, whereas the traditional motivation framework developed for reward-guided behavior emphasizes explicit choices (Niv et al., 2006; Schultz, 2006) (but see McClure et al., 2004). We introduce the idea that the motor system has its own motivation circuit, which operates analogously to but separately from explicit motivation. We suggest that this "motor motivation" works implicitly (i.e., outside of awareness) and governs automatic and spontaneous behavior, such as the speed of a reaching movement.

The concept of motor motivation may not only account for bradykinesia but may also help explain the natural speed of movements. Several models of trajectory control predict movement trajectories by optimizing various cost terms (Todorov, 2004), which in some cases include an "effort penalty" term that penalizes movements with greater energy expenditure (Todorov and Jordan, 2002). An energy-related term reflects the intuition that, all else being equal, many movements do not appear to be made as fast and as accurately as possible. This term can take various forms, and $|\bar{a}|$ and average arm power are plausible terms for models that include an energy-related cost. A limitation of these models is that they do not predict MT. Conversely, our recent model predicts $\mathrm{MT}$ but does not take energy cost into account (Tanaka et al., 2006).

In light of these models, we investigated why patients with $\mathrm{PD}$ select a longer MT than controls, when they could move faster without a loss in accuracy or trajectory quality. Our results suggest that patients are more sensitive to the energetic cost of a movement for a given accuracy. Specifically, we found that patients were less likely than control subjects to select a speed that met criterion on any given movement. The measure of the probability of appropriate speed selection $\left(N_{c}\right)$ correlated directly with $|\bar{a}|$, a measure of energetic cost. The slope of this correlation $\left(S_{N}\right)$ was significantly steeper in patients than controls, which indicates a shift in sensitivity of the cost-reward trade-off. The high correlation between $S_{N}$ and the UPDRS motor score is strong evidence that $S_{N}$ is a biologically meaningful measure. The UPDRS motor score is a reliable marker of loss of striatal dopaminergic terminals (Morrish et al., 1996), which supports our proposed role for striatal dopamine level as the signal encoding motor motivation, captured by $S_{N}$.

The concept of motor motivation extends a recently proposed role of striatal dopamine in "energizing" action (Niv et al., 2006). In this model, dopamine signals in the dorsolateral striatum influence response vigor, measured as frequency of responses. We propose that striatal dopamine also "energizes" action in a more literal sense, namely by assigning a value to the energetic cost of moving. A critical feature of our result, namely, the patients' reduction in motor vigor in the absence of reductions in speed or accuracy, fits the authors' suggestion that the energizing action of dopamine is outcome independent (Niv et al., 2006). Our results also provide indirect anatomical support for this model, which assigns the "action-energizing" role to dopamine activity in the dorsolateral striatum (as opposed to an "action-directing" role for dopamine in the dorsomedial striatum), because the lateral striatum is the site of earliest manifestation of dopamine deficiency in PD (Kish et al., 1988). A "neuroeconomics" scheme involving reward, cost, and utility functions was recently used to describe the control of force magnitude and duration in a weightlifting task (Kording et al., 2004). An energizing role for dopamine in such a framework would link naturally to previous characterizations of bradykinesia as a manifestation of "insufficient 
motor energy" (Hallett, 1990). In this framework, the slope $S_{N}$ reflects the underlying value of a fast accurate movement (cost/ benefit ratio): how much effort (speed) a movement of a given accuracy is "worth."

Our finding that PD patients had a normal speed-accuracy trade-off is consistent with some previous studies (Montgomery and Nuessen, 1990; Majsak et al., 1998) but contradicts others (Sheridan and Flowers, 1990; Phillips et al., 1994), which described a larger increase in endpoint variability when $P D$ patients moved faster. One possible explanation is that patients in the previous studies made movements either in reaction-time mode (they were instructed to move when a tone sounded) or under external pacing. When healthy subjects are forced to make a visually guided movement before they are ready, the resulting movement is inaccurate, reflecting incomplete specification of the motor plan (Hening et al., 1988; Favilla et al., 1989). The increased motor preparation time that has been described in PD (Brumlik and Boshes, 1966; Evarts et al., 1981; Hallett, 1990), and confirmed in our study, could thus lead to a degradation of the motor plan, resulting in movement inaccuracy, when patients attempt to move as soon as possible rather than when ready. Allowing subjects in our study to make each movement only when ready allowed full, and thus more accurate, formulation of the motor plan before each movement. Patients in the previous studies were also clinically more affected than our subjects. It should be emphasized, however, that the demonstration of movement variability in more advanced PD would not invalidate our findings: we found bradykinesia in the absence of higher variability, suggesting it is a primary abnormality. A correlation between bradykinesia and movement variability in later disease stages would then simply reflect their parallel, but independent, evolution as disease progresses.

\section{Conclusions}

The present study provides mechanistic insight into both the pathophysiology of bradykinesia in PD and motor planning in general. The explanation for bradykinesia suggested by our study is that patients with PD, a dopamine-depleting disease, have a higher probability of moving slowly because of a specific distortion of speed selection mechanisms: movements with a lower energy expenditure are favored although a repertoire of normal movements is available. This explanation for bradykinesia can plausibly be generalized to healthy subjects: movement speed is determined not only by the speed-accuracy trade-off but also by an implicit value assigned to movement energy cost. This value is manifested as response intensity (i.e., "movement vigor"). Thus, just as dopamine from the ventral tegmental area projects to the frontal cortex to modulate the cost/reward structure behind conscious decisions (Schultz, 1998; Graybiel, 2005), we propose that dopamine from the substantia nigra to the striatum carries an analogous signal for "motor" motivation.

\section{References}

Berardelli A, Rothwell JC, Thompson PD, Hallett M (2001) Pathophysiology of bradykinesia in Parkinson's disease. Brain 124:2131-2146.

Brumlik J, Boshes B (1966) The mechanism of bradykinesia in parkinsonism. Neurology 16:337-344.

England AC, Schwab RS (1961) Parkinson's syndrome. N Engl J Med 265:785-792.

Evarts EV, Teravainen H, Calne DB (1981) Reaction time in Parkinson's disease. Brain 104:167-186.

Fahn S, Elton RL, and the members of the UPDRS Development Committee (1987) Unified Parkinson's disease rating scale. In: Recent developments in Parkinson's disease II (Fahn S, Marsden CD, Goldstein M, Calne DB, eds), pp 153-163. New York: MacMillan.
Favilla M, Hening W, Ghez C (1989) Trajectory control in targeted force impulses. VI. Independent specification of response amplitude and direction. Exp Brain Res 75:280-294.

Fitts PM (1954) The information capacity of the human motor system in controlling the amplitude of movement. J Exp Psychol 47:381-391.

Folstein MF, Folstein SE, McHugh PR (1975) “Mini-mental state.” A practical method for grading the cognitive state of patients for the clinician. J Psychiatr Res 12:189-198.

Graybiel AM (2005) The basal ganglia: learning new tricks and loving it. Curr Opin Neurobiol 15:638-644.

Hallett M (1990) Clinical neurophysiology of akinesia. Rev Neurol (Paris) 146:585-590.

Hallett M, Khoshbin S (1980) A physiological mechanism of bradykinesia. Brain 103:301-314.

Hatze H, Buys JD (1977) Energy-optimal controls in the mammalian neuromuscular system. Biol Cybern 27:9-20.

Hening W, Favilla M, Ghez C (1988) Trajectory control in targeted force impulses. V. Gradual specification of response amplitude. Exp Brain Res $71: 116-128$.

Jeannerod M (1986) Mechanisms of visuomotor coordination: a study in normal and brain-damaged subjects. Neuropsychologia 24:41-78.

Kish SJ, Shannak K, Hornykiewicz O (1988) Uneven pattern of dopamine loss in the striatum of patients with idiopathic Parkinson's disease. Pathophysiologic and clinical implications. N Engl J Med 318:876-880.

Kording KP, Fukunaga I, Howard IS, Ingram JN, Wolpert DM (2004) A neuroeconomics approach to inferring utility functions in sensorimotor control. PLoS Biol 2:e330.

Majsak MJ, Kaminski T, Gentile AM, Flanagan JR (1998) The reaching movements of patients with Parkinson's disease under self-determined maximal speed and visually cued conditions. Brain 121:755-766.

McClure SM, York MK, Montague PR (2004) The neural substrates of reward processing in humans: the modern role of FMRI. Neuroscientist $10: 260-268$.

Montgomery Jr EB, Nuessen J (1990) The movement speed/accuracy operator in Parkinson's disease. Neurology 40:269-272.

Morrish PK, Sawle GV, Brooks DJ (1996) An [18F]dopa-PET and clinical study of the rate of progression in Parkinson's disease. Brain 119:585-591.

Niv Y, Joel D, Dayan P (2006) A normative perspective on motivation. Trends Cogn Sci 10:375-381.

Niv Y, Daw ND, Joel D, Dayan P (2007) Tonic dopamine: opportunity costs and the control of response vigor. Psychopharmacology (Berl) 191:507-520.

Phillips JG, Martin KE, Bradshaw JL, Iansek R (1994) Could bradykinesia in Parkinson's disease simply be compensation? J Neurol 241:439-447.

Reinsch CH (1967) Smoothing by spline functions. Numerische Math 10:177-183.

Sainburg RL, Ghez C, Kalakanis D (1999) Intersegmental dynamics are controlled by sequential anticipatory, error correction, and postural mechanisms. J Neurophysiol 81:1045-1056.

Schmidt RA, Zelaznik H, Hawkins B, Frank JS, Quinn JT (1979) Motoroutput variability: a theory for the accuracy of rapid motor acts. Psychol Rev 86:415-451.

Schultz W (1998) Predictive reward signal of dopamine neurons. J Neurophysiol 80:1-27.

Schultz W (2006) Behavioral theories and the neurophysiology of reward. Annu Rev Psychol 57:87-115.

Sheridan MR, Flowers KA (1990) Movement variability and bradykinesia in Parkinson's disease. Brain 113:1149-1161.

Silverman BW (1986) Density estimation for statistics and data analysis. London: Chapman and Hall.

Sokal RR, Rohlf FJ (1981) Biometry: the principles and practice of statistics in biological research, Ed 2. New York: Freeman.

Tanaka H, Krakauer JW, Qian N (2006) An optimization principle for determining movement duration. J Neurophysiol 95:3875-3886.

Todorov E (2004) Optimality principles in sensorimotor control. Nat Neurosci 7:907-915.

Todorov E, Jordan MI (2002) Optimal feedback control as a theory of motor coordination. Nat Neurosci 5:1226-1235.

Winter DA (1990) Biomechanics and motor control of human movement. New York: Wiley.

Woodworth RS (1899) The accuracy of voluntary movement. Psychol Rev 3 [Suppl 13]:1-119. 